\title{
The "white globe appearance" (WGA): a novel marker for a correct diagnosis of early gastric cancer by mag- nifying endoscopy with narrow-band imaging (M-NBI)
}

Authors

Institutions
Hisashi Doyama' ${ }^{1}$ Naohiro Yoshida', Sho Tsuyama², Ryosuke Ota' ${ }^{1}$, Yasuhito Takeda', Hiroyoshi Nakanishi', Kunihiro Tsuji', Kei Tominaga', Shigetsugu Tsuji', Kenichi Takemura', Shinya Yamada', Kazuyoshi Katayanagi², Hiroshi Kurumaya ${ }^{2}$, Akinori Iwashita ${ }^{3}$, Kenshi $\mathrm{Yao}^{4}$

Institutions are listed at the end of article. submitted

25. September 2014 accepted after revision 15. October 2014

\section{Bibliography}

DOI http://dx.doi.org/

10.1055/s-0034-1391026

Published online: 14.1.2015

Endoscopy International Open

2015; 03: E120-E124

(C) Georg Thieme Verlag KG

Stuttgart · New York

E-ISSN 2196-9736

\section{Corresponding author}

\section{Hisashi Doyama, MD, PhD}

Department of

Gastroenterology

Ishikawa Prefectural Central

Hospital

2-1 Kuratukihigashi, Kanazawa

Ishikawa 920-8530

Japan

Fax: +81-76-238-2377

doyama.134@ipch.jp
Background and study aims: Although magnifying endoscopy with narrow-band imaging (M$\mathrm{NBI}$ ) is useful for the diagnosis of gastric mucosal lesions, differentiating between early cancer (EC) and low grade adenoma (LGA) remains a challenge. During M-NBI examination, we have noted the presence of a small, white lesion with a globular shape underneath cancerous gastric epithelium, and have termed this endoscopic finding the "white globe appearance" (WGA). The aim of this study was to determine whether or not the WGA could be an endoscopic marker for distinguishing EC from LGA.

Methods: We retrospectively analyzed both the M-NBI scans and resected specimens of a total of 111 gastric lesions from 95 consecutive patients. Our main outcome was a difference in the prevalence of the WGA in EC and LGA.

\section{Introduction}

$\nabla$

Because magnifying endoscopy with narrowband imaging (M-NBI) can clearly visualize both the gastric subepithelial microvascular architecture and the microsurface structure [1], it is useful for the diagnosis of gastric mucosal lesions [2-4]. However, differentiating between cancer and adenoma remains a challenge $[3,5]$. Followup without endoscopic treatment for low grade adenoma (LGA) is permitted because the risk of progression for LGA to gastric cancer is relatively low [6].

During M-NBI examination, we have noted the presence of a small, white lesion with a globular shape ( $<1 \mathrm{~mm}$ ) underneath cancerous gastric epithelium. It is invisible under nonmagnifying endoscopy. Additionally, this finding is more clearly visualized with NBI than with white-light imaging and is rarely detected in noncancerous lesions. We have termed this endoscopic finding the "white globe appearance" (WGA). By careful histological investigation, some of the WGA visu-
Results: The prevalence of the WGA in EC and LGA was $21.5 \%$ (20/93) and $0 \%(0 / 18)$, respectively $(P$ $=0.039$ ). The sensitivity, specificity, positive predictive value, and negative predictive value for differentiating between EC and LGA, according to the presence of the WGA, were 21.5, 100, 100, and $19.8 \%$, respectively.

Conclusion: A positive WGA in a suspicious lesion on M-NBI would be an adjunct to the M-NBI diagnosis of possible EC because the specificity and positive predictive value of the WGA for differentiating between EC and LGA were extremely high. The WGA could be a novel endoscopic marker for differentiating between EC and LGA.

alized with M-NBI was found to correspond to intraglandular necrotic debris (IND) within markedly dilated neoplastic glands, suggesting it as a possible histological marker specific for cancer [7]. Accordingly, this study was undertaken to determine the accuracy of the WGA as an endoscopic marker for gastric cancer.

\section{Methods}

\section{$\nabla$}

Study design and patients

This observational study was conducted at a single tertiary referral center in Japan, as part of the Strengthening the Reporting of Observational Studies in Epidemiology (STROBE) program [8]. In accordance with the Declaration of Helsinki, the institutional review board of Ishikawa Prefectural Central Hospital approved this study, and written informed consent was obtained from all subjects.

We retrospectively reviewed both the M-NBI images and resected specimens of a total of 122 gas- 
tric lesions from 106 consecutive patients, who had undergone preoperative M-NBI examination and lesion resection by endoscopic submucosal dissection (ESD) between July 2013 and January 2014 at our hospital. ESD was principally indicated for gastric cancer under the following conditions: differentiated intramucosal adenocarcinoma without ulceration regardless of size; differentiated intramucosal adenocarcinoma with ulceration and $\leq 3$ $\mathrm{cm}$ in size; and undifferentiated intramucosal adenocarcinoma without ulceration and $\leq 2 \mathrm{~cm}$ in size. These conditions were determined by preoperative biopsy and/or endoscopy. Therefore, 20 gastric adenomas were followed up without endoscopic treatment. Baseline characteristics and endoscopic and histopathological data were reviewed by means of medical records. We excluded 3 lesions for which histopathological diagnoses based on the resected specimens were benign and 8 lesions for which MNBI findings were at a low magnification or out of focus-leaving 111 lesions from 95 patients suitable for final analysis ( $\bullet$ Fig. 1).

\section{Endoscopy system and setting}

We used an upper gastrointestinal magnifying endoscope (GIFH260Z, Olympus Medical Systems, Tokyo, Japan), a video processor (EVIS LUCERA Olympus CV-260SL, Olympus Medical Systems), and a light source (EVIS LUCERA Olympus CLV-260SL, Olympus Medical Systems). The structure enhancement of the endoscopic video processor was set to B-mode level 8 for M-NBI. The color mode was fixed at level 1 . To obtain stable endoscopic images at maximal magnification, a black, soft hood (MAJ-1990, Olympus Medical Systems) was mounted at the tip of the endoscope prior to examination.

\section{Endoscopic definitions and investigation of WGA}

The WGA was defined as a small, white lesion with a globular shape $(<1 \mathrm{~mm})$ present underneath the gastric epithelium and identified during M-NBI examination ( $\bullet$ Fig. 2). Criteria for a positive WGA were less intense peripheral brightness than in the center (reflecting its globular shape) and the presence of overlying microvessels, because the WGA lies underneath the gastric epithelium and the subepithelial microvessels. The presence or absence of the WGA in early cancer (EC), LGA, or non-neoplastic background mucosa (BM) was retrospectively assessed using MNBI images taken at maximal magnification rate by an experienced endoscopist (H. D.) who was not aware of the histology. If the WGA inside a neoplasm was recognized with a demarcation line between the neoplastic lesion and the surrounding mucosa, this distribution was termed "marginal."

\section{Histopathological investigation}

Endoscopically resected specimens were extended on boards with pins and fixed in $10 \%$ formalin for 24 hours. After fixation, all resected specimens were cut into 2 - to 3-mm thick longitudinal slices. These were embedded in paraffin and stained with hematoxylin-eosin. Postoperative histopathological diagnosis was performed by two pathologists and the results were doublechecked for all cases. Histopathological diagnoses were made with reference to the revised Vienna classification [9]. For the purposes of this study, we defined the revised Vienna category 3 as LGA and the revised Vienna categories 4 and 5 as EC, reclassifying all lesions into LGA and EC groups. The EC group was subclassified into differentiated (intestinal) and undifferentiated (diffuse) types.

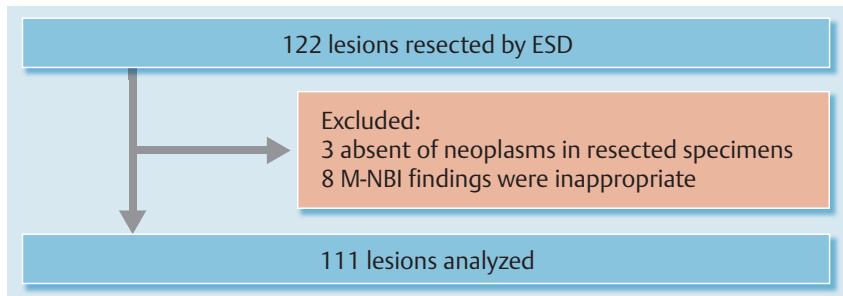

Fig. 1 Flowchart of the review and analysis. ESD, endoscopic submucosal dissection; M-NBI, magnifying endoscopy with narrow-band imaging.

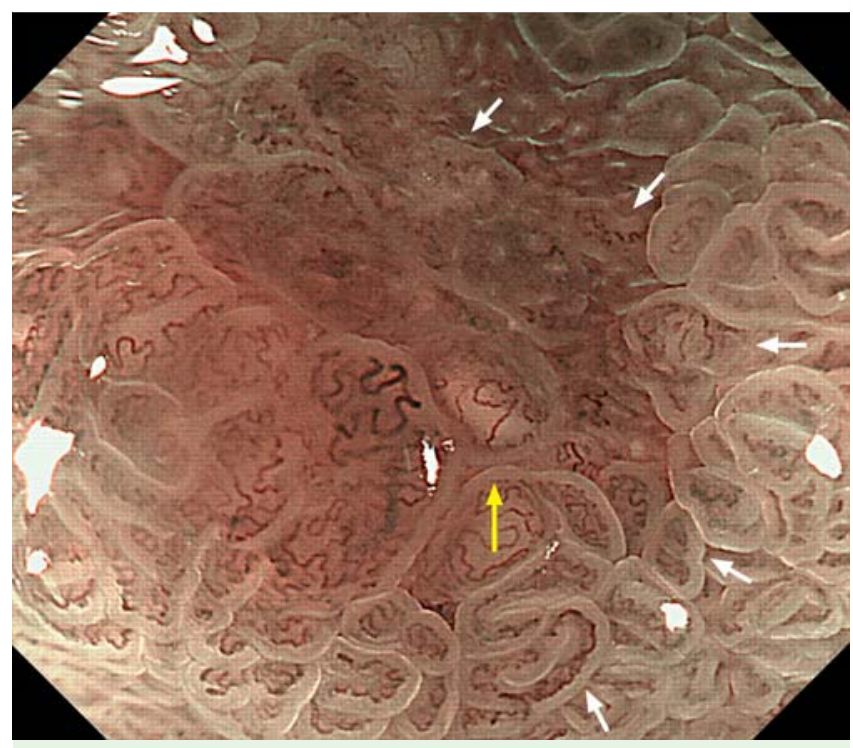

Fig. 2 Representative endoscopic image of the white globe appearance (WGA) visualized by magnifying endoscopy with narrow-band imaging (M-NBI) with maximal magnification. The WGA inside cancerous mucosa (yellow arrow) was located close to the demarcation line between the cancerous mucosa and the surrounding mucosa (white arrows), indicative of "marginal distribution." The WGA features were a whitish color less intense at the lesion's periphery than in the center (reflecting its globular shape), and the presence of overlying microvessels. The WGA was approximately $0.5 \mathrm{~mm}$ in size. The lesion size was determined by observation using a visual field of approximately $3.4 \mathrm{~mm}$ with maximal magnification as a guide, with the black hood mounted on the endoscope.

\section{Histological definitions and investigation of IND}

IND was defined as eosinophilic material with necrotic epithelial fragments within the lumen of a dilated gland ( $\mathbf{F i g . 3 a - c}$ ). There was segmental necrosis of the glandular lining, characterized by cytoplasmic vacuolization and dark nuclei [7]. The presence or absence of IND was retrospectively assessed using resected specimens by an experienced pathologist (S.T.) who was unaware of the endoscopic findings.

\section{Outcome measurements}

Outcomes were: (1) a difference in the prevalence of the WGA in EC and LGA; (2) the prevalence of the WGA in BM; (3) a correlation between the presence of the WGA and IND; and (4) clinicopathological characteristics of EC associated with the WGA.

\section{Statistical analysis}

Continuous variables were compared using Student's $t$-test. Categorical variables were compared using the $X^{2}$ or Fisher's exact test when the expected values were less than 5. $P<0.05$ was consid- 

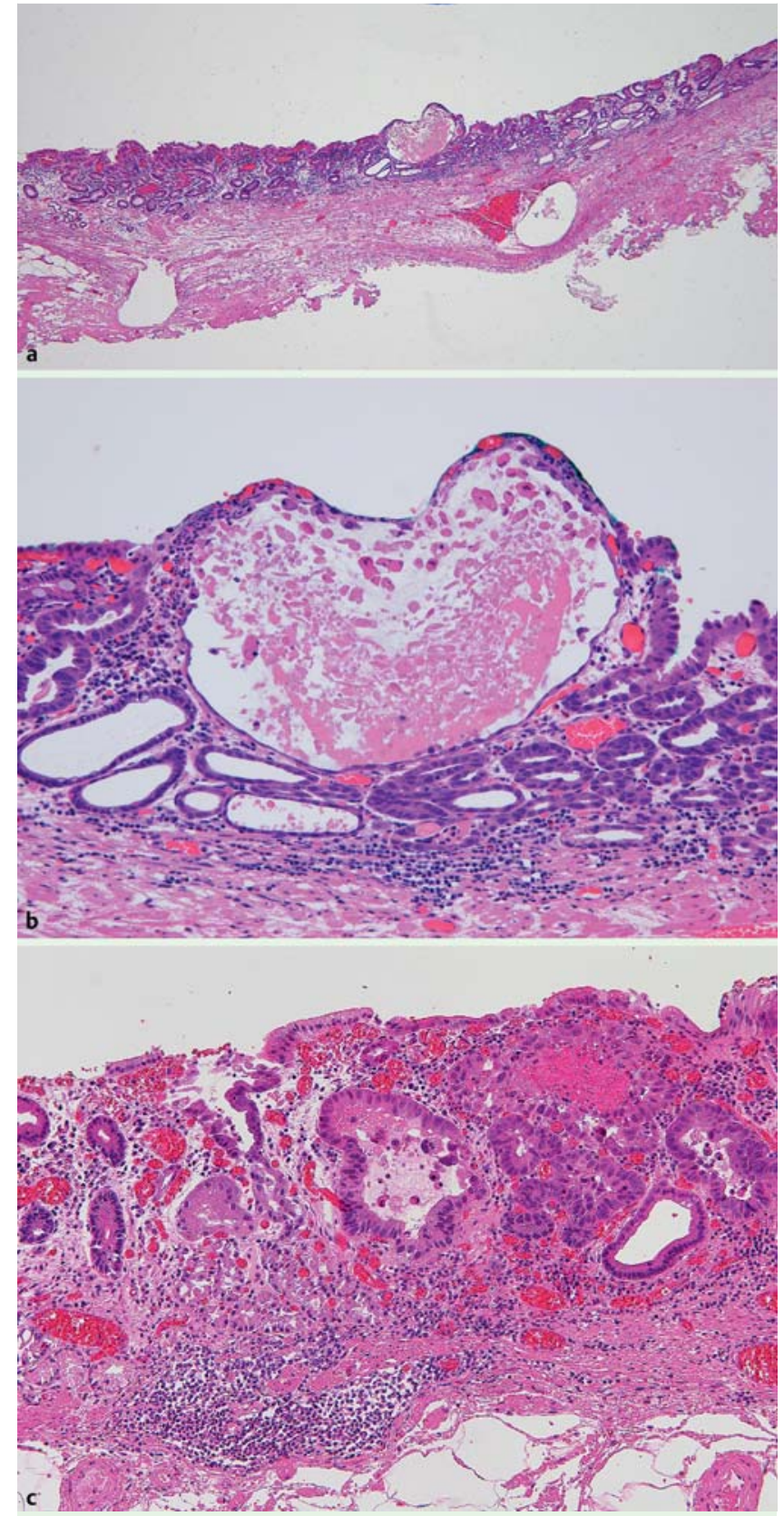

Fig. 3 Representative histological images of intraglandular necrotic debris (IND). a The IND within markedly dilated neoplastic glands was present underneath the cancerous gastric epithelium. By careful histological investigation, this IND measuring $0.43 \mathrm{~mm}$ was proven to be identical to the white globe appearance (WGA). b Magnified view of Fig. $\mathbf{3}$ a. $\mathbf{c}$ The IND within dilated neoplastic glands was present near a lateral margin of the cancer. However, the IND was not located just underneath the cancerous gastric epithelium but in the deeper part of the lamina propria in the cancerous tissue. This IND was $0.18 \mathrm{~mm}$ in size.

ered statistically significant. All analyses were performed using statistical software (JMP 11, SAS Institute Inc., Cary, NC, USA).
Table 1 Clinicopathological characteristics of 111 gastric lesions from 95 patients.

\begin{tabular}{|c|c|c|}
\hline \multicolumn{3}{|l|}{ Sex, no. (\%) } \\
\hline Male & 73 & (76.8) \\
\hline Female & 22 & $(23.2)$ \\
\hline Age, mean, y & 71 & \\
\hline Tumor size, mean, mm & 13.7 & \\
\hline \multicolumn{3}{|l|}{ Histological type, no.(\%) } \\
\hline EC & 93 & (83.8) \\
\hline Differentiated & 89 & $(80.2)$ \\
\hline Undifferentiated & 4 & (3.6) \\
\hline LGA & 18 & $(16.2)$ \\
\hline \multicolumn{3}{|l|}{ Macroscopic type, no. (\%) } \\
\hline 0 -IIc & 52 & $(46.8)$ \\
\hline 0 -IIa & 49 & $(44.1)$ \\
\hline $0-11 \mathrm{~b}$ & 7 & (6.3) \\
\hline $0-I$ & 3 & (2.7) \\
\hline \multicolumn{3}{|l|}{ Tumor location, no. (\%) } \\
\hline Upper third & 19 & (17.1) \\
\hline Middle third & 57 & (51.4) \\
\hline Lower third & 35 & (31.5) \\
\hline \multicolumn{3}{|c|}{ Ulcerative finding, no. (\%) } \\
\hline Positive & 5 & $(4.5)$ \\
\hline Negative & 106 & (95.5) \\
\hline \multicolumn{3}{|c|}{ Tumor depth of EC, no. (\%) } \\
\hline Mucosal & 86 & (92.5) \\
\hline Submucosal & 7 & (7.5) \\
\hline
\end{tabular}

Abbreviations: EC, early cancer; LGA, low grade adenoma.

\section{Results}

$\nabla$

\section{Clinicopathological characteristics}

There were 73 males and 22 females, with an average age of 71 (range $50-89$ ). The mean tumor diameter was $13.7 \mathrm{~mm}$ (range 1-41 mm). The histological types were LGA in 18 lesions and EC in 93 lesions. According to the Paris endoscopic classification [10], the macroscopic types were 0-IIc in 52 lesions, 0 -IIa in 49 lesions, 0-IIb in 7 lesions, and 0-I in 3 lesions. The tumor locations were upper third in 19 lesions, middle third in 57 lesions, and lower third in 35 lesions. There were 5 lesions with ulcerative findings. The tumor depths of EC were mucosal in 86 lesions and submucosal in 7 lesions ( $\bullet$ Table $\mathbf{1})$.

\section{Prevalence of the WGA in EC, LGA, and BM}

The prevalence of the WGA in EC and LGA was $21.5 \%(20 / 93)$ and $0 \%(0 / 18)$, respectively $(P=0.039)$ ( $\bullet$ Table 2$)$. The sensitivity, specificity, positive predictive value, and negative predictive value for differentiating between EC and LGA according to the presence of the WGA were $21.5 \%$ (95\% confidence interval [CI] 13.7 $31.2 \%$ ), $100 \%$ (95\%CI $84.7-100 \%$ ), $100 \%$ (95\%CI $86.1-100 \%$ ), and $19.8 \%$ (95\%CI $12.2-29.4 \%$ ), respectively. In addition, the prevalence of the WGA in BM was $0 \%(0 / 111)$.

\section{Correlation between WGA and IND}

When we investigated the WGA and IND separately, 6 of 20 WGA-positive lesions were negative for IND, and 33 of 91 WGA-negative lesions were positive for IND ( $\bullet$ Table 3$)$. The sensitivity, specificity, positive predictive value, and negative predictive value for the presence of IND according to the presence of the WGA were $29.8 \%$ (95\%CI $17.3-44.9 \%$ ), $90.6 \%$ (95\% CI $80.7-96.5 \%), 70 \%$ (95\%CI $45.7-88.1 \%$ ), and $63.7 \%$ (95\%CI $53.0-73.6 \%)$, respectively. 
Table 2 Prevalence of the WGA in EC and LGA.

\begin{tabular}{|lllllll|}
\hline & n & Present & Absent & \multicolumn{2}{c|}{ P value } \\
\hline EC & 93 & 20 & $(21.5)$ & 73 & $(78.5)$ & 0.039 \\
\hline LGA & 18 & 0 & $(0)$ & 18 & $(100)$ & \\
\hline
\end{tabular}

Abbreviations: EC, early cancer; LGA, low grade adenoma; WGA, white globe appearance.

Sensitivity, $21.5 \%$ (95\% confidence interval [CI] 13.7-31.2\%); specificity, 100\% (95\%Cl 84.7-100\%); positive predictive value, 100\% (95\%Cl 86.1 - 100\%); negative predictive value, $19.8 \%$ ( $(95 \%$ Cl $12.2-29.4 \%)$.

Data are expressed as number (\%).

Table 3 Histological prevalence of IND according to the presence of WGA.

\begin{tabular}{|c|c|c|c|c|c|}
\hline & & \multicolumn{4}{|c|}{ IND } \\
\hline & & \multicolumn{2}{|c|}{ Positive $(n=47)$} & \multicolumn{2}{|c|}{ Negative $(n=64)$} \\
\hline \multirow[t]{2}{*}{ WGA } & Positive $(n=20)$ & 14 & (70) & 6 & (30) \\
\hline & Negative $(n=91)$ & 33 & $(36.3)$ & 58 & (63.7) \\
\hline
\end{tabular}

Abbreviations: IND, intraglandular necrotic debris; WGA, white globe appearance.

Data are expressed as number (\%).

\begin{tabular}{|c|c|c|c|c|c|}
\hline & Present & & Absent & & $P$ value \\
\hline Tumor size, mean, mm & 15.8 & & 13.8 & & 0.40 \\
\hline Macroscopic type, no. (\%) & & & & & 0.86 \\
\hline 0 -IIC & 11 & $(21.6)$ & 40 & $(78.4)$ & \\
\hline 0 -IIa & 7 & $(21.2)$ & 26 & $(78.8)$ & \\
\hline $0-I I b$ & 2 & $(28.6)$ & 5 & (71.4) & \\
\hline $0-I$ & 0 & (0) & 2 & $(100)$ & \\
\hline Tumor location, no.(\%) & & & & & 0.0046 \\
\hline Upper third & 6 & (35.3) & 11 & $(64.7)$ & \\
\hline Middle third & 14 & $(28.6)$ & 35 & $(71.4)$ & \\
\hline Lower third & 0 & (0) & 27 & $(100)$ & \\
\hline Ulcerative finding, no. (\%) & & & & & 0.065 \\
\hline Positive & 3 & $(60)$ & 2 & $(40)$ & \\
\hline Negative & 17 & (19.3) & 71 & $(80.7)$ & \\
\hline Histological type, no. (\%) & & & & & 1.00 \\
\hline Differentiated & 19 & (21.3) & 70 & $(78.7)$ & \\
\hline Undifferentiated & 1 & $(25)$ & 3 & $(75)$ & \\
\hline Tumor depth, no. (\%) & & & & & 1.00 \\
\hline Mucosal & 19 & $(22.1)$ & 67 & (77.9) & \\
\hline Submucosal & 1 & (14.3) & 6 & $(85.7)$ & \\
\hline
\end{tabular}

Abbreviations: EC, early cancer; WGA, white globe appearance.

\section{Clinicopathological characteristics of EC with WGA}

There was no correlation between the presence of the WGA and tumor size, macroscopic type, ulcerative findings, histological type, or depth $(P>0.05)$. The prevalence of the WGA in the lower third of the stomach was significant $(P=0.0046)$ ( $\odot$ Table 4). Especially in 0-IIa, it was difficult to distinguish EC from LGA in non-ulcerative, differentiated, and mucosal cancers; the prevalence of the WGA was $17.9 \%$ (5/28), similar to that of other ECs. The average number of WGA in 20 lesions with WGA was 2.3 (range 1-5). Also, 46 (95.8\%) of the 48 WGA were judged as marginal distribution. There was no correlation between the number of WGA and tumor size, macroscopic type, ulcerative findings, histological type, or depth $(P>0.05)$.

\section{Discussion}

The WGA was evident in EC lesions but not in LGA. The specificity and positive predictive value of the WGA for differentiating between EC and LGA were extremely high, although the sensitivity and negative predictive value were low. We have often experienced cancerous lesions with low confidence prediction, as demonstrated by a prospective multicenter M-NBI study [3]. Certainly, the presence of the WGA adds to the specificity of M-NBI diagnoses with low confidence prediction. A positive WGA in a suspicious lesion on M-NBI would be an adjunct to an M-NBI diagnosis of possible EC. Watanabe et al. reported that no IND was detected in cases of LGA, and was detected in only 1 of 52 cases in Vienna category 1 or 2 [7]. We also detected no WGA in LGA or BM. The WGA might show promise in differentiating between cancer and gastritis.

We had predicted that the WGA would correlate exactly with the finding of IND just underneath the gastric epithelium; it seeming 
logical that IND would be detected on histology more frequently than the WGA being detected with M-NBI. However, 30\% of WGA-positive lesions were negative for IND. This may be due to two factors. First, there was a 1-week to 1-month interval between preoperative M-NBI examination and the ESD procedure. We have experienced discrepancies between visualization of WGA on M-NBI examinations that were performed on different days. We speculate that these same discrepancies apply to the histological presence of IND. Second, the size of the IND was too small to be detected in the histopathological sections cut into 2 to 3-mm thicknesses. On the other hand, approximately 35\% WGA-negative lesions were positive for IND. This also may be due to two factors. First, the IND was present not only just underneath the gastric cancerous epithelium but also within deep layers of the lamina propria in the cancerous tissue, as shown in - Fig. 3c. All IND cannot be identified if we examine from the surface of the mucosa using M-NBI. Second, since the horizontal extent of neoplasms needs to be determined during the preoperative $\mathrm{M}-\mathrm{NBI}$ examinations, we mainly photographed its margins. Accordingly, we may have missed any WGA located in a nonmarginal distribution with respect to the neoplasm.

Although differentiated and submucosal cancers showed the highest incidence of IND in a report by Watanabe et al. [7], there was no correlation between the presence of the WGA and histological type or depth in our study. We suggest that the reason is that the cancers included in this study were limited to intramucosal or submucosal lesions that showed microinvasion into the submucosa because we recruited patients who were candidates for ESD.

We also found that the WGA in EC tended to demonstrate a predominantly marginal distribution. By electron microscopy, there is a spectrum of apoptotic-necrotic phenomena in ordinary (nonmucinous) adenocarcinomas of the gastrointestinal tract, ranging from crypt lumen apoptosis (mainly because of apoptosis of adenocarcinoma cells) to "dirty necrosis" and IND (mainly because of necrosis-like cell death) up to tissue necrosis [11]. The apoptotic-necrotic phenomena may predominate near the margin of EC. The newly developed optical technology of M-NBI might allow endoscopic visualization of the spectrum of apoptotic-necrotic phenomena in EC presenting with the WGA.

Our study had several limitations. First, it was retrospective. Second, we found a number of WGA-negative lesions that were positive for IND. As we focused on the margins of neoplasms during preoperative M-NBI, we may have underestimated the prevalence of the WGA. Its prevalence in BM may also have been underestimated. Third, the number of submucosal and undifferentiated cancers was small because the subjects of this study were limited to the patients who had been candidates for ESD. Fourth, the prevalence of the WGA in focal gastritis is unclear. It is as yet un- clear whether the presence of the WGA can make a contribution to the improvement of real-time diagnostic performance in clinical practice. We have begun a prospective study to avoid these limitations (UMIN 000013650).

In conclusion, M-NBI made it possible to visualize the WGA in the stomach. Like IND, which is a possible histological marker specific for EC, the WGA could be a novel endoscopic marker for differentiating between EC and LGA.

\section{Competing interests: None}

\section{Institutions}

${ }^{1}$ Department of Gastroenterology, Ishikawa Prefectural Central Hospital, Kanazawa, Japan

2 Department of Diagnostic Pathology, Ishikawa Prefectural Central Hospital, Kanazawa, Japan

3 Department of Pathology, Fukuoka University Chikushi Hospital, Chikushino, Japan

${ }^{4}$ Department of Endoscopy, Fukuoka University Chikushi Hospital, Chikushino, Japan

\section{References}

1 Muto M, Katada C, Sano Y et al. Narrow band imaging: a new diagnostic approach to visualize angiogenesis in the superficial neoplasia. Clin Gastroenterol Hepatol 2005; 3: 16-20

2 Yao K, Anagnostopoulos GK, Ragunath K. Magnifying endoscopy for diagnosing and delineating early gastric cancer. Endoscopy 2009; 41: $462-467$

3 Yao K, Doyama H, Gotoda T et al. Diagnostic performance and limitations of magnifying narrow-band imaging in screening endoscopy of early gastric cancer: a prospective multicenter feasibility study. Gastric Cancer 2014; 17: 669-679

4 Ezoe $Y$, Muto $M$, Uedo $N$ et al. Magnifying narrowband imaging is more accurate than conventional white-light imaging in diagnosis of gastric mucosal cancer. Gastroenterology 2011; 141: 2017-2025

5 Miwa $K$, Doyama $H$, Ito $R$ et al. Can magnifying endoscopy with narrow band imaging be useful for low grade adenomas in preoperative biopsy specimens? Gastric Cancer 2012; 15: 170-178

6 Yamada H, Ikegami M, Shimoda T et al. Long-term follow-up study of gastric adenoma/dysplasia. Endoscopy 2004; 36: 390-396

7 Watanabe $Y$, Shimizu $M$, Itoh $T$ et al. Intraglandular necrotic debris in gastric biopsy and surgical specimens. Ann Diagn Pathol 2001; 5: $141-147$

8 von Elm E, Altman DG, Egger $M$ et al. Strengthening the reporting of observational studies in epidemiology (STROBE) statement: guidelines for reporting observational studies. BMJ 2007; 335: 806-808

9 Schlemper RJ, Riddell RH, Kato Y et al. The Vienna classification of gastrointestinal epithelial neoplasia. Gut 2000; 47: 251-255

10 Participants in the Paris Workshop. The Paris endoscopic classification of superficial neoplastic lesions: esophagus, stomach and colon: November 30 to December 1, 2002. Gastrointest Endosc 2003; 58: 3-43

11 Caruso RA. Tumor necrosis with special reference to autophagic cell death (self-cannibalism) and xeno-cannibalism in gastric cancer: our experience and review of the literature. In: Tompkins MB, ed. Gastric cancer research trends. New York: Nova; 2007: 273-306 